\title{
Hipertensão arterial na infância: uma revisão de literatura
}

\author{
Arterial hypertension in childhood: a literature review \\ Hipertensión arterial en la infancia: revisión de la literatura
}

Recebido: 27/08/2021 | Revisado: 01/09/2021 | Aceito: 05/09/2021 | Publicado: 09/09/2021

\author{
Rafael dos Santos da Conceição \\ ORCID: https://orcid.org/0000-0002-9654-8403 \\ Universidade Estadual de Roraima, Brasil \\ E-mail: rafaelrivaille@gmail.com \\ Iara Leão Luna de Souza \\ ORCID: https://orcid.org/0000-0002-5309-2979 \\ Universidade Estadual de Roraima, Brasil \\ Centro Universitário Estácio da Amazônia, Brasil \\ E-mail: iaraluna@uerr.edu.br
}

\begin{abstract}
Resumo
A Hipertensão Arterial (HA) é uma condição clínica caracterizada por elevação sustentada dos níveis pressóricos, comumente investigada na população adulta. Entretanto, a hipertensão arterial na infância e adolescência, definida por valores de pressão arterial sistólica e/ou diastólica iguais ou superiores ao percentil 95 para sexo, idade e altura em três ou mais ocasiões diferentes é raramente investigada. Nesse contexto, evidências recentes apontam uma correlação entre o aumento da pressão arterial (PA) na infância e adolescência, principalmente a partir de 6 anos, com o crescimento da epidemia de obesidade nessa faixa populacional. Ademais, preconiza-se que as crianças acima dos 3 anos tenham sua PA aferida pelo menos uma vez ao ano, e em todas as visitas médicas se eles apresentarem pelo menos um dos fatores de risco para hipertensão. Logo, as diretrizes da prática clínica recomendam que um diagnóstico seja estabelecido quando se encontrarem valores idênticos de PA em três visitas separadas, sendo tais valores iguais ou superiores ao percentil 95. Como as evidências apontam que a HA constitui seus pilares nos primeiros anos de vida do indivíduo, atestando que níveis elevados de pressão arterial nessa faixa etária são fortes preditores dessa condição em adultos, esse é o foco desta revisão.
\end{abstract}

Palavras-chave: Hipertensão; Obesidade pediátrica; Pressão arterial; Infância.

\begin{abstract}
Arterial Hypertension (AH) is a clinical condition characterized by a sustained increase in blood pressure levels, commonly investigated in the adult population. However, arterial hypertension in childhood and adolescence, defined by systolic and/or diastolic blood pressure values equal to or greater than the 95th percentile for sex, age and height on three or more different occasions is rarely investigated. In this context, recent evidence points to a correlation between the increase in blood pressure (BP) in childhood and adolescence, especially from the age of 6 , with the growth of the obesity epidemic in this population range. In addition, it is recommended that children above 3 years old have their BP checked at least once a year, and in all medical visits if they present at least one of the risk factors for hypertension. Therefore, clinical practice guidelines recommend that a diagnosis be established when identical BP values are found on three separate visits, with such values being equal to or greater than the 95th percentile. As the evidence points out, $\mathrm{AH}$ constitutes its pillars in the first years of life of the individual, attesting that high blood pressure levels in this age group are strong predictors of this condition in adults, this is the focus of this review.
\end{abstract}

Keywords: Hypertension; Pediatric obesity; Blood pressure; Childhood.

\section{Resumen}

La Hipertensión Arterial (HA) es una condición clínica caracterizada por un aumento sostenido de la presión arterial, comúnmente investigado en la población adulta. Sin embargo, la hipertensión en la infancia y la adolescencia, definida por valores de presión arterial sistólica y/o diastólica iguales o superiores al percentil 95 para el sexo, la edad y la altura en tres o más ocasiones diferentes, rara vez se investiga. En este contexto, evidencia reciente apunta a una correlación entre el aumento de la presión arterial (PA) en la infancia y la adolescencia, especialmente después de los 6 años, con el crecimiento de la epidemia de obesidad en este grupo de población. Además, se recomienda que a los niños mayores de 3 años se les mida la PA al menos una vez al año, y en todas las visitas médicas si presentan al menos uno de los factores de riesgo de hipertensión. Por tanto, las guías de práctica clínica recomiendan que se establezca un diagnóstico cuando se encuentren valores de PA idénticos en tres visitas separadas, siendo dichos valores iguales o superiores al percentil 95 del individuo, acreditando que los niveles de hipertensión arterial en este grupo de edad son fuertes predictores de esta afección en adultos, este es el enfoque de esta revisión.

Palabras clave: Hipertensión; Obesidad pediátrica; Presión arterial; Infancia. 


\section{Introdução}

A Hipertensão Arterial (HA) em adultos é uma condição clínica caracterizada por elevação sustentada dos níveis pressóricos, com o valor sistólico igual ou maior 140 e/ou valor diastólico igual ou maior a $90 \mathrm{mmHg}$. Além disso, associa-se a eventos como morte súbita, Acidente Vascular Encefálico (AVE) e Doença Renal Crônica (DRC), fatal e não fatal (Brasil, 2016).

A respeito da hipertensão arterial na infância e adolescência, que inclui os indivíduos dentro da faixa de idade de 0 aos 17 anos, valores de pressão arterial sistólica e/ou diastólica iguais ou superiores ao percentil 95 para sexo, idade e altura em três ou mais ocasiões diferentes, são os fatores considerados para que se confirme tal condição (Brasil, 2019). Pesquisas de cunho epidemiológico que tratam sobre o objeto de estudo deste trabalho têm sido importante fonte de subsídios, fornecendo dados consistentes de que, de forma invariável, crianças com níveis pressóricos elevados tendem a mantê-los em faixas elevadas ao longo da vida, de forma que torna possível frisar que a hipertensão arterial do adulto se inicia na infância (Salgado \& Carvalhaes, 2003).

Geralmente, não há uma causa atribuível à expressão de HA em um indivíduo, sendo este tipo de hipertensão classificada como essencial ou primária. No entanto, uma pequena parcela desses casos está relacionada a causas determináveis, as quais incluem um espectro amplo de doenças. Dentre as principais causas, destacam-se problemas renais e endócrinos, seguidos por problemas cardíacos, neurológicos, psicológicos e exógenos, como o abuso de drogas e exposição a substâncias nocivas (Brasil, 2014).

Concomitantemente, a obesidade e o sobrepeso foram comprovadamente associados ao aparecimento e manutenção de valores de PA elevados em diversas faixas etárias, mostrando ainda que, à medida que o sobrepeso era tratado, os níveis pressóricos eram igualmente reduzidos (Silva, 2005).

Atualmente, pelo fato da não incorporação da aferição de pressão arterial (PA) em exames de rotina de pacientes pediátricos, muitos casos acabam sendo subnotificados ou tardiamente diagnosticados, expondo o paciente a consequências tardias de tal condição clínica (Silva et al., 2018). Tal aferição, em crianças, segue a mesma linha do que é preconizado em adultos, devendo ser realizada em crianças maiores de 3 anos pelo menos uma vez ao ano, estando a medição em indivíduos abaixo desta idade em um espectro de situações específicas (Weaver, 2019).

No que tange à terapêutica, o tratamento para a HA pediátrica pode inicialmente envolver ou não o uso de medicamentos, devendo, portanto, ser analisado de acordo com o quadro individual de cada paciente (Brasil, 2016). Quando necessário, o tratamento farmacológico deve ser iniciado com um inibidor de enzima conversora de angiotensina (ECA), um bloqueador do receptor de angiontensina II, um bloqueador de canal de cálcio de ação prolongada ou um diurético tiazídico (Flynn et al., 2017).

Diante desse contexto, essa revisão tem por objetivo elucidar as principais causas da hipertensão na infância, a importância de seu rastreio precoce e as variáveis existentes para o diagnóstico desta condição clínica em crianças.

\section{Metodologia}

Este estudo é constituído por uma revisão integrativa da literatura, e as etapas incluíram a identificação do tema e seleção da hipótese ou questão de pesquisa para a elaboração da revisão; o estabelecimento de critérios para inclusão e exclusão de estudos; a definição das informações a serem extraídas dos estudos selecionados e sua categorização; a avaliação dos estudos incluídos na revisão através de critérios pré-estabelecidos; a interpretação dos resultados e a redação da revisão integrativa (Sousa et al., 2018).

As bases de dados que foram utilizadas nesta revisão incluem a Scientific Electronic Library Online (SciELO), a US National Library of Medicine National Institutes of Health (PUBMED) e a Literatura Científica e Técnica da América Latina e 
Caribe (LILACS), além de Diretrizes e Manuais das Sociedades Brasileiras de Cardiologia e Pediatria.

Os descritores utilizados, em português e inglês, foram hipertensão arterial, hipertensão arterial na infância, pressão arterial alta em crianças, obesidade infantil e hipertensão, fatores de risco para hipertensão e saúde da criança e do adolescente.

Os artigos foram selecionados de acordo com o título e seu escopo principal, utilizando-se daqueles que tem o tema de estudo em destaque. Entretanto, artigos que apresentaram definições obsoletas acerca de determinados conceitos foram excluídos do estudo.

\section{Resultados e Discussão}

Há uma tendência ao aumento da PA com a idade, pois os vasos sanguíneos naturalmente reduzem sua luz e perdem a elasticidade com o passar dos anos, favorecendo o aparecimento da hipertensão. Entretanto, é observado que o risco do desenvolvimento de tal condição em crianças e adolescentes está semelhantemente aumentado, devido ao grande número de indivíduos nessa faixa etária que possuem sobrepeso ou obesidade (Eua, 2020).

Antigamente, a hipertensão primária era considerada como um diagnóstico de exclusão na população pediátrica. Porém, evidências recentes sugerem taxas crescentes de hipertensão primária correspondentes à epidemia de obesidade. A hipertensão primária é o tipo mais comum de hipertensão em crianças mais velhas, ao passo que a hipertensão secundária continua sendo a etiologia mais frequente de PA elevada em lactentes e jovens crianças (Benenson, Frederick \& Porter, 2020). Em um estudo que incluiu a participação de 423 crianças com história clínica de níveis pressóricos elevados, constatou-se que 65\% (275) desses indivíduos possuíam HA, 37\% proveniente de causas secundárias e 28\% de causas impossíveis de serem estabelecidas (hipertensão essencial ou primária); ou seja, a hipertensão por causas secundárias foi relatada em $57 \%$ das 275 crianças com hipertensão (Gupta et al., 2015).

Os distúrbios renais são as etiologias mais comuns de hipertensão secundária infantil. Segundo dados dos Estados Unidos, especificamente, condições parenquimatosas renais e anormalidades estruturais (como glomerular, anomalias tubulares e intersticiais) respondem por 34 a $79 \%$ dos casos de hipertensão secundária, enquanto distúrbios renovasculares (como estenose da artéria renal) são encontrados em 12 a 13\% dos pacientes (Flynn et al., 2017).

Nesse âmbito, os principais fatores de risco para a HA são a idade, sexo, obesidade ou sobrepeso, etnia e hábitos de vida desregulados e pouco saudáveis, como o consumo excessivo de bebidas alcoólicas e do sal, sedentarismo, tabagismo, além de outros fatores relacionados à história familiar e à predisposição genética. Ao se promover a explanação de tais fatores, postula-se que níveis pressóricos elevados são mais comuns em afro-americanos que em brancos, asiáticos e hispânicos, sendo homens abaixo de 50 anos mais propensos a desenvolverem HA do que as mulheres; após os 50 anos, entretanto, essa ordem é invertida e as mulheres lideram o número de casos (Silva, Oliveira \& Pierin, 2016).

Há evidências de que a hipertensão arterial primária, fator de risco independente para as duas causas mais frequentes de morte em adultos no Brasil (cardiopatia isquêmica e acidente vascular cerebral), constitui seus pilares na infância ou adolescência, atestando que níveis elevados de pressão arterial nessa faixa etária são preditores de HA em adultos (Souza et al., 2010). Além disso, um estudo constatou que adolescentes com PA elevada progrediam para hipertensão a uma taxa de $7 \%$ ao ano. Logo, pacientes jovens com HA provavelmente enfrentarão um processo acelerado de envelhecimento vascular, o que pode aumentar ainda mais seus níveis pressóricos com o passar da idade (Falkner et al., 2008).

O peso e o índice de massa corporal (IMC) são as variáveis que apresentam a mais forte correlação com a PA na infância e adolescência, principalmente a partir de seis anos (Neto et al., 2018). No Brasil, dados de 2019 do Sistema de Vigilância Alimentar e Nutricional (Sisvan), apontam que três de cada 10 crianças entre 5 a 9 anos atendidas pelo Sistema Único de Saúde (SUS) estão acima do peso, o que resulta em 4,4 milhões de indivíduos. Do total de crianças, 16\% (2,4 milhões) estão com sobrepeso, $8 \%$ (1,2 milhão) com obesidade e 5\% (755 mil) com obesidade grave. Abaixo de 5 anos, são 
observadas 15,9\% com excesso de peso (Brasil, 2020). Estudos conduzidos pela Organização Mundial de Saúde (OMS) projetam que, em 2025, haverá 75 milhões de crianças obesas ao redor do mundo, fator que, somado a outros fatores genéticos e ambientais, pode desencadear a expressão de níveis pressóricos elevados. Adicionalmente, dados recentes da Europa demonstram que os números de casos de HA aumentam ainda mais entre crianças com sobrepeso ou obesidade, alcançando um índice de 22\% de prevalência (Burrello et al., 2018).

Os mecanismos fisiopatológicos que favorecem o desenvolvimento de HA no indivíduo obeso são complexos e ainda não foram completamente elucidados. Tais mecanismos estão dispostos na Figura 1, que correlaciona as principais características fisiológicas dos indivíduos obesos ao aumento de seus níveis pressóricos. Dentre as alterações, destacam-se as hemodinâmicas sistêmicas e renais, ativação do sistema nervoso simpático (SNS) e do sistema renina-angiotensinaaldosterona, além da hiperinsulinemia compensatória; esta última é decorrente da resistência à insulina presente em indivíduos obesos, principalmente naqueles que apresentam excesso de gordura na região do tronco, promovendo ativação do SNS e reabsorção tubular de sódio, que é apontada como o mecanismo inicial básico do aumento da PA de obesos (Marchi-Alves et al., 2010).

Ainda sobre os fatores que relacionam o excesso de peso com a maior suscetibilidade de desenvolvimento da hipertensão, a leptina, hormônio encontrado em concentrações elevadas em indivíduos obesos, demonstrou relação com o aumento da PA em pelo menos algumas populações humanas, por ocasionar o aumento do tônus simpático nos rins, nas adrenais e no coração. A ativação do SNS mediada por insulina e leptina pode ser visualizada como um mecanismo recrutado nesses pacientes para estabilizar o peso e restaurar o balanço energético, favorecendo a termogênese por estimulação simpática e aprimorando a produção de energia; porém, como consequência, também ocorre aumento da pressão arterial (Landsberg et al., 2013).

Figura 1. Correlação da obesidade com o desenvolvimento da hipertensão arterial. SNS: sistema nervoso simpático; DC: débito cardíaco; RPT: resistência periférica total; RAAS: sistema renina-angiotensina-aldosterona; ANGII: angiotensina.

II.

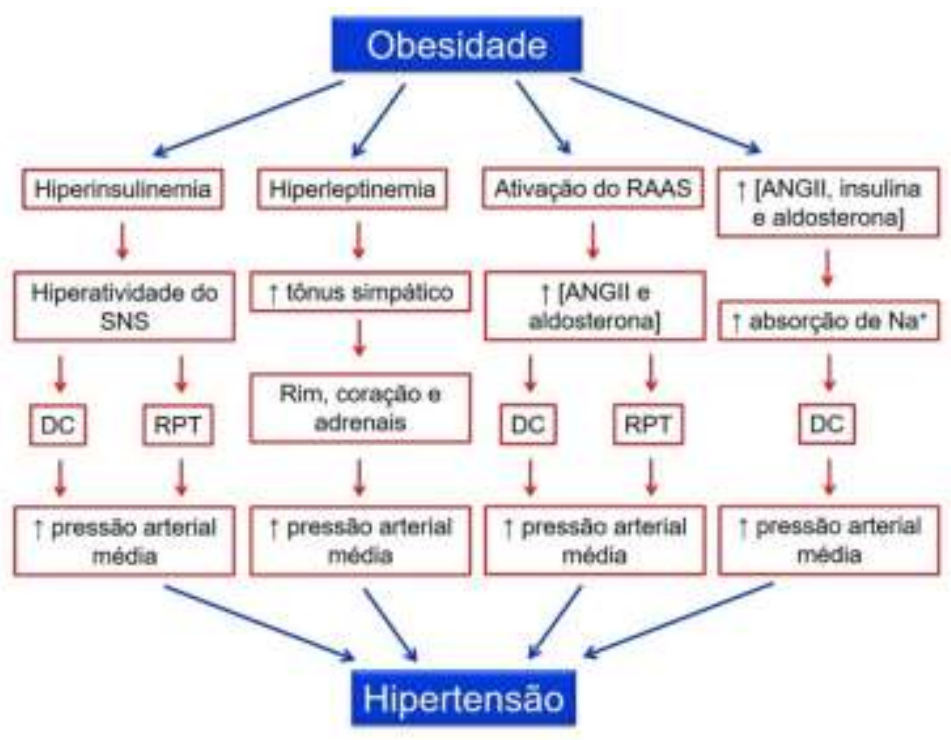

Fonte: Autores.

Dessa forma, nota-se que elevados níveis de gordura corporal são responsáveis por alterações importantes nos mecanismos que regem o funcionamento adequado de vários sistemas do organismo, desgastando-os de forma precoce, o que, provavelmente, irá repercutir na qualidade e expectativa de vida desses indivíduos (Ferreira \& Aydos, 2010). 


\subsection{Técnicas de aferição da PA}

Preconiza-se que todas as crianças acima dos 3 anos tenham sua PA aferida pelo menos uma vez ao ano, e em todas as visitas médicas se eles apresentarem pelo menos um dos fatores de risco para hipertensão (como obesidade, doença renal, diabetes) ou se eles utilizarem medicamentos que possam persistentemente elevar a pressão arterial (esteroides, estimulantes do sistema nervoso central, hormonais contraceptivos), estando a aferição em indivíduos de idade inferior em um espectro de situações específicas. Assim, bebês prematuros ou com baixo peso ao nascer, portadores de doenças cardíacas, renais ou que tenham passado por transplante de órgãos ou medula óssea, são exemplos de candidatos que devem passar pelo processo de aferição da PA desde os primeiros anos de vida. Os métodos de aferição utilizados em crianças e adolescentes, incluindo os neonatos, são postulados e serão descritos a seguir.

A aferição da PA na criança segue o mesmo modelo do que é preconizado em adultos. O ideal é que a criança esteja sentada ou deitada, tranquila, descansada por mais de 5 minutos, com a bexiga vazia e sem ter praticado exercícios físicos há pelo menos 60 minutos. O paciente deve estar deitado ou sentado, com pernas descruzadas, pés apoiados no chão, dorso recostado na cadeira e relaxado; com o braço ao nível do coração, sendo preferencial o braço direito, para ser comparável com as tabelas padrão e evitar falsas medidas baixas no braço esquerdo no caso de coarctação da aorta. O braço deve estar na altura do coração, apoiado, com a palma da mão voltada para cima e as roupas não devem garrotear o membro (Brasil, 2019; Sousa et al., 2018).

É necessário medir a circunferência do braço para a escolha do manguito, logo, $1^{\circ}$ passo: medir a distância do acrômio ao olécrano; $2^{\circ}$ passo: identificar o ponto médio da distância entre o acrômio e o olécrano; $3^{\circ}$ passo: medir a circunferência do braço nesse ponto médio. A partir dessa medida, seleciona-se o manguito adequado para a medida, que deve cobrir $40 \%$ da largura e 80 a 100\% do comprimento. Não se deve avaliar especificamente pela faixa etária do paciente, mas sim pela medida da circunferência do braço. Em serviços pediátricos, deve-se ter disponibilidade completa de manguitos, devido à extensa faixa etária e variação de tamanho da população que é atendida (desde recém-nascidos até adolescentes obesos) (Brasil, 2019; Sousa et al., 2018).

Após a escolha do manguito adequado, $4^{\circ}$ passo: colocar o manguito, sem deixar folgas, 2 a $3 \mathrm{~cm}$ acima da fossa cubital; $5^{\circ}$ passo: centralizar o meio da parte compressiva do manguito sobre a artéria braquial; $6^{\circ}$ passo: estimar o nível da pressão arterial sistólica (PAS) pela palpação do pulso radial; $7^{\circ}$ passo: palpar a artéria braquial na fossa cubital e colocar a campânula ou o diafragma do estetoscópio sem compressão excessiva; $8^{\circ}$ passo: inflar rapidamente até ultrapassar 20 a 30 mmHg o nível estimado da PAS obtido pela palpação; $9^{\circ}$ passo: proceder à deflação lentamente (velocidade de 2 $\mathrm{mmHg} /$ segundo); $10^{\circ}$ passo: determinar a PAS pela ausculta do primeiro som (fase I de Korotkoff) e, após, aumentar ligeiramente a velocidade de deflação; $11^{\circ}$ passo: determinar a PA diastólica (PAD) no desaparecimento dos sons (fase $\mathrm{V}$ de Korotkoff); $12^{\circ}$ passo: auscultar cerca de 20 a $30 \mathrm{mmHg}$ abaixo do último som para confirmar seu desaparecimento e depois proceder à deflação rápida e completa; $13^{\circ}$ passo: se os batimentos persistirem até o nível zero, determinar a PAD no abafamento dos sons (fase IV de Korotkoff) e anotar valores da PAS/PAD/zero; $14^{\circ}$ passo: anotar os valores exatos sem arredondamentos, lembrando que, pelo método auscultatório, o intervalo entre os valores marcados no manômetro é de 2 mmHg (Brasil, 2019; Sousa et al., 2018).

Não obstante, caso neonatos se enquadrem no grupo daqueles que devem ter sua PA aferida antes dos 3 anos, vários métodos estão disponíveis para que se realize tal medição, o que inclui métodos intra-arteriais diretos, usando cateteres internos, bem como medidas indiretas, que são as técnicas oscilométrica, que utiliza aparelhos eletrônicos, e auscultatória, que utiliza o aparelho aneroide juntamente com o estetoscópio. No consultório, no entanto, o método oscilométrico é a técnica normalmente usada pelo menos até que o bebê seja capaz de cooperar com a determinação manual da pressão arterial (que também depende da capacidade do indivíduo que irá realizar a aferição de obter PA auscultatória em lactentes e crianças 
pequenas) (Brasil, 2019; Sousa et al., 2018).

Valores normativos para PA neonatal e infantil geralmente têm sido determinados no quadrante superior do braço direito com o bebê em decúbito dorsal, sendo que abordagem semelhante deve ser seguida no ambulatório. Assim como acontece com crianças mais velhas, o tamanho do manguito é importante na obtenção de leituras precisas da pressão arterial em neonatos. O comprimento da bexiga do manguito deve circundar 80 a 100\% do braço circunferência; uma bexiga com uma relação de circunferência largura-braço de 0,45 a 0,55 é recomendada Além disso, o dispositivo de aferição utilizado deve ser validado em neonatos e programado para ter um valor inicial de inflação apropriado para lactentes (geralmente $\leq 120 \mathrm{mmHg}$ ). A auscultação torna-se tecnicamente viável quando o braço do bebê é grande o suficiente para o menor manguito disponível para os dispositivos auscultatórios. É melhor fazer medições quando a criança está em um estado calmo; múltiplas leituras podem ser necessárias se a primeira leitura for elevada, semelhante à técnica recomendada para crianças mais velhas (Brasil, 2019; Sousa et al., 2018).

\subsection{Classificação da PA em crianças e adolescentes}

No que tange à classificação, para que se tenha um parâmetro correto para avaliação da PA, deve-se levar em conta a idade do paciente que será examinado. Dessa forma, a classificação da HA seguindo os percentis para sexo, idade e altura, é utilizada em indivíduos com até 13 anos. Adicionalmente, a definição de hipertensão em crianças e adolescentes é baseada na distribuição normativa da PA em crianças saudáveis. A PA normal é definida como PAS e PAD com percentil <90\% para sexo, idade e altura. A hipertensão é definida como PAS ou PAD com percentil $\geq 95^{\circ}$ para sexo, idade e altura em pelo menos três ocasiões distintas. Os níveis médios de PAS ou PAD, com percentil $\geq 90$, mas $<$ percentil 95 , foram designados como "alto normal" e considerados uma indicação de risco aumentado para o desenvolvimento de hipertensão (EUA, 2004).

A partir dos 13 anos, os níveis de PA em adultos já podem ser adotados, o que objetiva alinhar as diretrizes pediátricas com as de adultos e facilitar a conduta terapêutica e a transição de adolescentes mais velhos com PA elevada e HA. Assim, a classificação da PA conforme a idade está como demonstrada na Tabela 1. Ressalta-se, no entanto, que esse corte arbitrário em 13 anos deve ser analisado individualmente. Considera-se que, além da idade, deve ser levado em conta o estágio puberal da criança, só devendo ser utilizado esse valor a partir de 13 anos se o indivíduo já estiver na puberdade (Brasil, 2019). 
Tabela 1. Classificação da pressão arterial de acordo com a faixa etária. PA: pressão arterial; P90: percentil 90; P95: percentil 95.

\begin{tabular}{cc}
\hline Crianças de 1 a 13 anos de idade & Crianças com idade $\geq 13$ anos de idade \\
Normotensão & Normotensão \\
PA $<$ P90 para sexo, idade e altura & PA $<120 /<80 \mathrm{mmHg}$ \\
Pressão arterial elevada & Pressão arterial elevada \\
PA $\geq$ P90 e $<$ P95 para sexo, idade e altura ou PA & PA $120 /<80 \mathrm{mmHg}$ a PA $129 /<80 \mathrm{mmHg}$ \\
$120 / 80 \mathrm{mmHg}$ mas $<$ P95 (o que for menor) & \\
Hipertensão estágio 1 & Hipertensão estágio 1 \\
PA $\geq$ P95 para sexo, idade e altura até $<$ P95 + & PA $130 / 80$ ou até $139 / 89$ \\
$12 \mathrm{mmHg}$ ou PA 130/80 ou até 139/89 (o que for & \\
menor) & Hipertensão estágio 2 \\
Hipertensão estágio 2 & PA $\geq$ entre $140 / 90$ \\
PA $\geq$ P95 $+12 \mathrm{mmHg}$ para sexo, idade ou altura & \\
ou PA $\geq$ entre 140/90 (o que for menor) &
\end{tabular}

Fonte: Brasil (2019).

Para classificar a pressão arterial de uma criança, ainda são necessárias as tabelas de percentil de altura (disponíveis em literatura pediátrica) e de percentil de pressão arterial, que são baseadas no The Fourth Report on the Diagnosis, Evaluation, and Treatment of High Blood Pressure in Children and Adolescents, de 2004. Então, uma vez obtidos os valores de PAS e PAD do paciente, cruza-se tais valores com o seu percentil de altura, estando ainda de acordo com sua idade.

\subsection{Diagnóstico}

Entre e durante os encontros de saúde, a PA em crianças é altamente variável. Crianças ansiosas ou agitadas podem exibir uma pressão arterial elevada isolada conhecida como hipertensão do jaleco branco, que geralmente não reflete os verdadeiros valores. Diante disso, as diretrizes da prática clínica recomendam que um diagnóstico de hipertensão seja estabelecido quando se encontraram valores idênticos de PA em três visitas separadas, sendo tais valores iguais ou superiores ao percentil 95 para crianças menores de 13 anos, como já citado.

Leituras feitas por máquinas oscilométricas são comumente maiores em comparação aos valores obtidos por ausculta; portanto, leituras auscultatórias devem ser usadas para confirmar o diagnóstico de hipertensão (Flynn et al., 2017).

Em situações específicas, como suspeita de hipertensão do jaleco branco ou verificação da eficácia de terapia antihipertensiva, deve-se lançar mão da Monitorização Ambulatorial da Pressão Arterial (MAPA). A MAPA registra a PA em intervalos definidos (geralmente a cada 30 minutos) durante atividades diárias regulares e sono, sendo mais precisa do que baseado em leituras. Se disponível, a MAPA deve ser usada rotineiramente para o diagnóstico de hipertensão em crianças (Brasil, 2011).

$\mathrm{Na}$ investigação das causas, é muito importante que seja verificada a história clínica e realizado exame físico, ambos de forma detalhada e completa, na tentativa de identificar aspectos que possam sugerir causa secundária da HA. Algumas das causas secundárias, divididas por faixa etária, estão dispostas no Quadro 1, a seguir. 
Quadro 1. Causas mais frequentes de hipertensão arterial por faixa etária na infância e adolescência.

\begin{tabular}{|l|l|}
\hline \multicolumn{1}{|c|}{ Crianças de 1 a 13 anos de idade } & \multicolumn{1}{|c|}{ Crianças com idade $\geq 13$ anos de idade } \\
\hline Normotensão: PA < P90 para sexo, idade e altura & Normotensão: PA < 120/<80 mmHg \\
\hline $\begin{array}{l}\text { Pressão arterial elevada: PA } \geq \text { P90 e }<\text { P95 para } \\
\text { sexo, idade e altura ou PA 120/80 mmHg mas < P95 } \\
\text { (o que for menor) }\end{array}$ & $\begin{array}{l}\text { Pressão arterial elevada: PA 120/<80 mmHg a PA } \\
129 /<80 \mathrm{mmHg}\end{array}$ \\
\hline $\begin{array}{l}\text { Hipertensão estágio 1: PA } \geq \text { P95 para sexo, idade e } \\
\text { altura até < P95 + 12mmHg ou PA 130/80 ou até } \\
\text { 139/89 (o que for menor) }\end{array}$ & Hipertensão estágio 1: PA 130/80 ou até 139/89 \\
\hline $\begin{array}{l}\text { Hipertensão estágio 2: PA } \geq \text { P95 + 12mmHg para } \\
\text { sexo, idade ou altura ou PA } \geq \text { entre 140/90 (o que for } \\
\text { menor) }\end{array}$ & Hipertensão estágio 2: PA $\geq$ entre 140/90 \\
\hline
\end{tabular}

Fonte: Brasil (2019).

\subsection{Tratamento}

A terapêutica inicial do controle da hipertensão em crianças é inicialmente não medicamentosa, prezando-se por uma mudança no estilo de vida, que incentiva a prática de atividades físicas e a mudança nos hábitos alimentares.

\subsubsection{Tratamento não-farmacológico}

Terapias não farmacológicas que apoiam nutrição e peso saudáveis, bem como a realização de atividade física devem ser plenamente recomendadas a todas as crianças com pressão arterial elevada e hipertensão. O efeito benéfico a longo prazo de abordagens não farmacológicas fora demonstrado em um estudo prospectivo de 798 crianças que foram acompanhados por 20 anos. A resolução da hipertensão foi observada em $44 \%$ dos participantes envolvidos em estilos de vida saudáveis (Benenson, Frederick \& Porter, 2020).

Modificações na dieta, como redução da ingestão de sal, ingestão limitada de açúcar, e um aumento da ingestão de frutas frescas, vegetais, grãos integrais, peixe, aves, nozes, carnes vermelhas magras e laticínios com pouca gordura, estão dentro da chamada Dietary Approaches to Stop Hypertension (DASH) e permanecem a pedra angular do tratamento para crianças hipertensas.

A atividade física regular é eficaz na redução da pressão arterial em crianças. O Centro de Controle e Prevenção de Doenças, no Physical activity guidelines for school-aged children and adolescentes, de 2018, apoia pelo menos 60 minutos de atividade física moderada a vigorosa diariamente para todas as crianças de 6 a 17 anos.

A diretriz de prática clínica recomenda que crianças hipertensas tratadas não farmacologicamente devem ser acompanhadas a cada 3 a 6 meses, assim, a adesão às modificações no estilo de vida pode ser reforçada com educação e apoio, podendo-se excluir a necessidade de terapias farmacológicas conforme a evolução do quadro clínico. Além disso, a educação e o apoio contínuos dos familiares e amigos permanecem essenciais (Flynn et al., 2017).

\subsubsection{Tratamento farmacológico}

A terapêutica farmacológica deve ser iniciada para casos pediátricos com HA sintomática, HA secundária, presença de lesão de órgão-alvo (LOA), diabetes melitus (DM tipos 1 e 2), doença renal crônica (DRC) e HA persistente não responsiva 
à terapêutica não farmacológica. Os eventos adversos associados com a utilização dos agentes anti-hipertensivos em crianças e adolescentes têm-se mostrado, em geral, de grau menor, como dores de cabeça, tonturas e infecções das vias respiratórias superiores. A utilização de todas as classes de anti-hipertensivos parece segura, pelo menos, em curto prazo, entretanto, Burrello e col. (2018), em sua meta-análise sobre a eficácia das diferentes classes de medicamentos empregados no tratamento de HA pediátrica, apoiam o emprego comum de inibidores da ECA como a primeira escolha de tratamento.

Sugere-se que, na HA secundária, a escolha do anti-hipertensivo seja feita em consonância com o princípio fisiopatológico envolvido, levando em consideração as comorbidades presentes em cada caso clínico. Por exemplo, os betabloqueadores não cardiosseletivos são evitados em pessoas com doença reativa das vias aéreas, devido ao risco de broncoespasmo. Os inibidores da enzima conversora de angiotensina (IECA) e os bloqueadores dos receptores de angiotensina (BRA) são contraindicados durante a gestação, devido ao potencial para malformações fetais (Brasil, 2016).

\section{Conclusão}

A HA é uma doença comprovadamente relacionada aos hábitos de vida do indivíduo, fato que se agrava ainda mais no contexto de infância e adolescência, devendo, portanto, serem devidamente controlados e monitorados. Para tanto, o acesso à informação, a educação em saúde, é pilar indispensável para o controle da hipertensão em crianças, o que, inevitavelmente, também criará adultos saudáveis. Destaca-se a importância de medidas preventivas, como: redução do consumo de alimentos industrializados ricos em sódio, controle do peso, combate ao sedentarismo, e ingestão de frutas, verduras e legumes. É de suma importância que os fatores e causas envolvidos no aparecimento de HA na infância se façam conhecidos, bem como sinais, sintomas e outras situações que tornam a investigação profunda de tal condição obrigatória. Tal conhecimento também pode servir de subsídio para órgãos governamentais desenvolverem projetos que visem melhores hábitos alimentares e de combate ao sedentarismo, tendo em vista que o sobrepeso e obesidade são a principal causa da elevação persistente dos níveis pressóricos em crianças; além disso, medidas como essa poderiam estimular esse público a exercerem seu papel de modificadores de comportamento em casa e na comunidade a qual estão inseridos.

\section{Agradecimentos}

Os autores agradecem à Universidade Estadual de Roraima pelo apoio institucional.

\section{Referências}

Benenson, I., Frederick, A. W. \& Porter, S. (2020). Pediatric hypertension: A guideline update. The Nurse Practitioner. 45 (5), $16-23$.

Brasil (2011). V Diretrizes Brasileiras de Monitorização Ambulatorial da Pressão Arterial (MAPA). Arquivos Brasileiros de Cardiologia. 97 (3), 6-16.

Brasil (2014). Linha guia de hipertensão. Secretaria de Estado da Saúde do Paraná. Superintendência de Atenção à Saúde. (2a ed.).

Brasil (2016). $7^{\text {a }}$ Diretriz Brasileira de Hipertensão Arterial. Arquivos Brasileiros de Cardiologia. 107 (3), 1-103.

Brasil (2019). Hipertensão arterial na infância e na adolescência. Sociedade Brasileira de Pediatria - Departamento Científico de Nefrologia. Manual de Orientação.

Brasil (2020). Ministério da Saúde. Campanha para prevenir obesidade infantil. Agência Brasil, https://www.google.com/amp/s/agenciabrasil.ebc. com.br /saude/noticia/2019-11/saude-lanca-campanha-para-prevenir-obesidade-infantil\%3famp.

Burrello, J., Erhardt, E. M., Saint-Hilary, G., Veglio, F., Rabbia, F., Mulatero, P., Monticone, S. \& D’Ascenzo, F. (2018). Pharmacological treatment of arterial hypertension in children and adolescents: a network meta-analysis. Hypertension. 72(2), 306-313

EUA (2004). National High Blood Pressure Education Program Working Group on High Blood Pressure in Children and Adolescents. The Fourth Report on the Diagnosis, Evaluation, and Treatment of High Blood Pressure in Children and Adolescents. Pediatrics. 114 (2), $555-76$.

EUA (2020). National Institutes of Health. National Heart, Lung and Blood Institute (NHLBI). High Blood Pressure, Bethesda, EUA. https://www.nhlbi.nih.gov/health-topics/high-blood-pressure. 
Falkner, B., Gidding, S. S., Portman, R. \& Rosner, B. (2008). Blood pressure variability and classification of prehypertension and hypertension in adolescence. Pediatrics. 122 (2), 238-242. 10.1542/peds.2007-2776.

Ferreira, J. S. \& Aydos, R. D (2010). Prevalência de hipertensão arterial em crianças e adolescentes obesos. Ciências e Saúde Coletiva. 15 (1), 97-104. https://doi.org/10.1590/S1413-81232010000100015

Flynn, J. T., Kaelber, D. C., Baker-Smith, C. M., Blowey, D., Carroll, A. E., Daniels, S. R., Ferranti, S. D., Dionne, J. M., Falkner, B., Flinn, S. K., Gidding, S. S., Goodwin, C., Leu, M. G., Powers, M. E., Rea, C., Samuels, J., Simasek, M., Thaker, V. V. \& Urbina, E. M. (2017). Clinical practice guideline for screening and management of high blood pressure in children and adolescents. Pediatrics. 140 (3), e20171904.

Gupta-Malhotra, M., Banker, A., Shete, S., Hashmi, S. S., Tyson, J. E., Barratt, M. S., Hecht, J. T., Milewicz, D. M. \& Boerwinkle, E. (2015). Essential hypertension vs. Secondary hypertension among children. American Journal of Hypertension. 28 (1), 73-80.

Landsberg, L., Aronne, L. J., Beilin, L. J., Burke, V., Igel, L. I., Lloyd-Jones, D. \& Sowers, J. (2013). Obesity-related hypertension: pathogenesis, cardiovascular risk, and treatment: a position paper of The Obesity Society and the American Society of Hypertension. Journal of Clinical Hypertension (Greenwich). 15 (1),14-33. 10.1111/jch.12049

Marchi-Alves, L. M., Nogueira, M. S., Mendes, I. A. C. \& Godoy, S. (2010). Leptina, hipertensão arterial e obesidade: importância das ações de enfermagem. Acta Paulista de Enfermagem. 23 (2), 286-90. https://doi.org/10.1590/S0103-21002010000200021

Neto, J. O. R., Pinheiro, A. A. L., Mynssen, B. V. \& Sampaio, M. C. R. (2018). Obesidade como fator de risco para hipertensão em crianças e adolescentes. Revista da Escola de Ciências Médicas de Volta Redonda. 1, 41-44.

Salgado, C. M. \& Carvalhaes, J. T. (2003). Hipertensão arterial na infância. Jornal de Pediatria. 79, S115-S124.

Silva, A. C. (2005). Pressão arterial em crianças e adolescentes de Porto Alegre e sua associação com estado socioeconômico e com três marcadores de sobrepeso e obesidade - índice de massa corporal, espessura de pregas cutâneas e circunferência da cintura. Dissertação (Mestrado em Ciências Médicas: Nefrologia) - Universidade Federal do Rio Grande do Sul, Rio Grande do Sul.

Silva, A. D., Oliveira, J. A., Immich, I. C. \& Kratz, C. P. (2018). Hipertensão Arterial na Infância: Um Estudo de Revisão. Revista Interdisciplinar em Ciências da Saúde e Biológicas. 2 (1), 77-96.

Silva, S. S., Oliveira, S. F. \& Pierin, A. M (2016). Controle da hipertensão arterial em mulheres e homens: uma análise comparativa. Revista da Escola de Enfermagem da USP. 50 (1), 50-58.

Sousa, L. M., Firmino, C. F., Marques-Vieira, C. A., Severino, S. S. P. \& Pestana, H. C. F. C. (2018). Revisões da literatura científica: tipos, métodos e aplicações em enfermagem. Revista Portuguesa de Enfermagem de Reabilitação. 1 (1), 46-55. 10.33194/rper.2018.v1.n1.07.4391.

Souza, M. G., Rivera, I. R., Silva, M. A., \& Carvalho, A. C. C. (2010). Relação da obesidade com a pressão arterial elevada em crianças e adolescentes. Arquivos Brasileiros de Cardiologia. 94 (6), 714-19.

Weaver, D. J. (2019). Pediatric Hypertension: Review of Update Guidelines. Pediatric Review. 40 (7), 354-58. 